\title{
LA CULTURA ESTÉTICA COMO EJE TRANSVERSAL EN EL CURRICULO DE EDUCACIÓN BÁSICA PARA EL DESARROLLO DE COMPETENCIAS
}

\section{AESTHETIC CULTURE AS A CROSS-SHAPE IN THE CURRICULUM OF BASIC EDUCATION FOR THE DEVELOPMENT OF COMPETENCES}

\author{
Yamil De Haz Cruz ${ }^{1}$ \\ Oscar Vélez Mora ${ }^{1}$ \\ Mónica Parra Cruz ${ }^{2}$ \\ 1 Universidad de Guayaquil. angela.dehazc@ug.edu.ec \\ 2 Colegio Delta. Guayaquil.
}

\section{RESUMEN}

El propósito de este estudio es evaluar la eficacia de las actividades de la enseñanza como una de las tareas propias del docente y uno de los componentes del currículo enmarcados en el proceso enseñanza aprendizaje de la asignatura de Dibujo Artístico, como eje transversal del currículo de educación básica. Esta evaluación permitió comprobar si las experiencias de aprendizaje producen los resultados esperados y también si estos determinan los aspectos positivos y negativos del diseño curricular. Se utilizó el estudio de casos, fundamentado en el diseño descriptivo, por el análisis e interpretación de la naturaleza actual, y la composición o procesos de los fenómenos trabajados sobre realidades de hecho y su característica fundamental es la presentación correcta aplicado al método científico por presentar exactitud en su conceptualización siendo los hechos fácticos y por qué se vale de la verificación empírica utilizando las variables transversales como la cultura estética, guía didáctica para el desarrollo de competencias, por lo que se ha definido el desarrollo del dibujo Artístico como destreza adquirida para el apoyo de otras asignaturas, cuando el estudiante no domina su motricidad fina, tendría inconvenientes en las actividades dirigidas en el aula de clases limitando su manera de representar cualquier objeto, basados en esta realidad se generan cambios conceptuales y analíticos de su desenvolvimiento.

Palabras claves: cultura, estética, currículo y competencias.

\begin{abstract}
The purpose of this study is to evaluate the effectiveness of teaching activities as one of the teacher 's own tasks and one of the components of the curriculum within the teaching - learning process of the subject of Artistic Drawing, as a transversal axis of the curriculum of basic education. This evaluation allowed to verify if the learning experiences produce the expected results and also if they determine the positive and negative aspects of the curricular design. We used the case study, based on descriptive design, for the analysis and interpretation of the current nature, and the composition or processes of the phenomena worked on realities of fact and its fundamental characteristic is the correct presentation applied to the scientific method to be presented Accuracy in its conceptualization being the factual facts and why it uses the empirical verification using the transversal variables as the aesthetic culture, didactic guide for the development of competences, reason why has defined the development of the Artistic drawing as skill acquired for the Support of other subjects, when the student does not master his fine motor skills, would have disadvantages in the directed activities in the classroom limiting the way of representing any object, based on this reality are generated conceptual and analytical changes of its development.
\end{abstract}

Keywords: Culture, aesthetics, curriculum and skills.

Recibido: 12/04/2017;

Aceptado: 26/11/2017;

Publicado: 19/12/2017 


\section{Introducción}

En su acuerdo ministerial 1419, de fecha 01 09 -1997, el Ministerio de Educación dice: La inserción de los lenguajes artísticos en el plan de estudios de la escuela ecuatoriana reformada constituye una contribución fundamental en la formación de la personalidad del niño y del joven, estimulan y elevan la sensibilidad del estudiante, favorecen el desarrollo general de las facultades, contribuyen al mejor entendimiento de su persona y del mundo, enriquecen su acervo cultural y al expresarse dan significado a muchas inquietudes en su mundo interior.

Como lo manifestó Mario Tamayo y Tamayo en el Diccionario de la Investigación Científica en el 2005: " El marco teórico nos ayuda a precisar y organizar los elementos y contenidos en la descripción del problema ".

CORREA DE MOLINA, Cecilia (1999) dijo: EI docente debe estar seguro de que al comunicarse con sus estudiantes, exprese realmente lo que quiere expresar. La vaguedad e imprecisión en los mensajes que transmite, pueden producir miedo, inseguridad, rechazo, burla e incredulidad. El docente no sólo debe escuchar las palabras que dicen los estudiantes, sino escuchar las que él mismo dice.

Las variables con las que se elaboró este artículo fueron: desarrollo pedagógico, y desarrollo de las destrezas psicomotrices en los estudiantes, para que mejoren su actitud hacia el arte y la planificación correcta de las actividades artísticas dentro del aula de clase, teniendo en cuenta la previa capacitación docente y la formulación de una guía didáctica.

\section{Esta concepción tiene por esencia:}

- Origen social de los procesos psicológicos.

- Hace uso de la zona de desarrollo próximo para facilitar los aprendizajes del estudiante.

- Promueve el aprendizaje integral, razonamiento, afecto o acción del estudiante.

- Hace pedagogía humanista.

- Papel fundamental del lenguaje y los signos como mediadores.

- Aprendizaje como proceso. Humaniza a los agentes educativos, mediante la creatividad y criticidad, cooperación y compromiso.

- Exalta la libertad y la creación.

- Es de orientación democrática y flexible.

Según esta pedagogía deben respetarse los siguientes principios:

- Los retos y problemas a estudiar son tomados de la realidad.

- El tratamiento y búsqueda de la situación problemática se trabaja de manera integral.

- Aprovechamiento de la oportunidad de observar a los compañeros en la acción para revelar los procesos ideológicos implícitos.

- La evaluación es integral y dinámica.

- Papel esencial del docente.

- El profesor como mediador del aprendizaje debe:

- Conocer los intereses de los estudiantes y sus diferencias individuales (inteligencias múltiples).

- Conocer las necesidades evolutivas de cada uno de ellos.

- Conocer los estímulos de sus contextos: familiares, comunitarios, educativos y otros.

- Contextualizar las actividades.

\section{La Cultura como Definición}

La cultura es el conjunto de todas las formas, los modelos y los patrones explícitos o implícitos, a través de los cuales una sociedad regula el comportamiento de las personas que la conforman. En el texto Historia de la Filosofía del Dr. Francisco Morán Márquez (2005) dijo: La cultura surge de la vida individual de la realidad, de lo inmediato se eleva a la categoría de lo general y de lo universal, es un acto creador. Deberíamos que así la vida social es como las demás formas de cultura, se nos dan bajo la especie de la vida individual de lo inmediato lo que hoy recibimos ya ornado con sublimes aureolas, tuvo a su tiempo que estrecharse y encogerse para pasar por el corazón de un hombre. (pág. 199)

La UNESCO, en 1982 declaró: " que la cultura da al hombre la capacidad de reflexionar sobre sí mismo. Es ella la que hace de nosotros seres específicamente humanos, racionales, críticos y éticamente comprometidos (Declaración de México)

La cultura no es un fenómeno material: no consiste cosas, gente, conductas o emociones. Es más bien una organización de todo eso. Es la forma de las cosas que la gente tiene en su mente, sus modos de percibirlas, de relacionarlas o de interpretarlas.

Habría que asociar más estrechamente a los 
docentes a las decisiones relativas a la educación. La elaboración de los programas escolares y del material pedagógico deberá hacerse con la participación de los docentes en ejercicio, en la medida en que la evaluación del aprendizaje no se puede disociar de la práctica pedagógica. Igualmente, el sistema de administración escolar de inspección, y de evaluación del personal docente ganará mucho si este participa en el proceso de decisión. Jacques Delors. (1996)

Según el Informe de la UNESCO, en su informe de seguimiento de la EPT en el mundo, la Educación básica es el Conjunto de actividades educativas realizadas en contextos diferentes (formal, no formal e informal) y destinadas a satisfacer las necesidades educativas básicas. En el Marco de Acción de Dakar, esta expresión es sinónimo del programa general de la EPT. Asimismo, las clasificaciones normalizadas del CAD de la OCDE y de la ayuda utilizan una definición que engloba la educación de la primera infancia, la enseñanza primaria y los programas destinados a los jóvenes y adultos a fin de que adquieran competencias básicas para la vida diaria, incluida la alfabetización. Según la Clasificación Internacional Normalizada de la Educación (CINE), la educación básica abarca la enseñanza primaria (primera etapa de la educación básica) y el primer ciclo de la enseñanza secundaria (segunda etapa).

Por lo consiguiente esta forma parte fundamental del desarrollo de la niñez y adolescencia. El mundo de hoy demanda la formación integral de personas con la capacidad de desenvolverse en una forma fluida y con ese toque de armonía que le permita desenvolverse en distintos contextos, obteniendo la capacidad de tomar decisiones, de trabajar con otros, de comunicar sus ideas, y de gestionar sus acciones en virtud de sus metas personales, escolares y laborales, entre otras.

La formación integral de los estudiantes es una necesidad imperiosa que conlleva múltiples beneficios. Es en ese momento que el currículo toma una importancia muy valedera porque debe de ser un acto de innovación en sí mismo. Así lo ha patentado en los trabajos de diseño curricular en los que se ha involucrado recientemente Estévez Nenninger, conformante del Consejo Mexicano de Investigación Educativa proponiendo currículos cambiantes, con características auto-gestivas que provoquen una innovación constante.

En este contexto y en cuanto a nuestro tema, se constata que las disciplinas de carácter artístico y cultural aparecen en muchos países, subvaloradas respecto de otras, y afectadas también por una subvaloración social que, en general, orienta a los estudiantes de mejores puntajes a privilegiar otras disciplinas.

\section{Transcendencia de la Cultura Estética}

Según José Manuel Ubals Álvarez define esta categoría de la siguiente manera: La cultura estética es la categoría con la que se designa:

- Todo el sistema de relaciones emocionales, sensibles, figurativas y estético-educativas que establece el hombre en sus nexos conscientes con la naturaleza, la sociedad, el arte y el propio hombre.

- Que denota un nivel de aprehensión espiritual de la realidad en una especie de síntesis cualificadora de las conexiones inmanentes a la actividad sujeto-objetual, observando a lo objetual desde una perspectiva de fascinación, embeleso, éxtasis y seducción subsiguiente.

- Que logra que se operacionalice en el polígono que se revela cuando en el sistema de trabazones hombre-hombre, hombrenaturaleza, hombre-arte y hombre-sociedad se permite una construcción espiritual de la belleza.

Lo anteriormente citado nos permite comprender que en el mapa curricular de educación básica debe estar presente un currículo transversal, cuyos propósitos de enseñanza comunes estén dirigidos hacia la atención de problemas del mundo, y este caso como eje principal en la educación básica, generando una serie de valores como el aprecio a la democracia, derechos humanos, equidad de género, la igualdad en la ciudadanía, así como el respeto por pluralidad y diversidad, cuidado del ambiente y de uno mismo. Las artes son consideradas para la infancia como un lenguaje cercano y familiar. La educación artística que en otros medios toma el nombre de Cultura Estética, y como añadido un valor fundamental para el incremento del bienestar social, que es el hecho de promover el desarrollo de personas vitales, felices y motivadas, que tomen iniciativas, accedan a sus propias decisiones, así como el que sean capaces de tomar iniciativas y de hacer sus propios descubrimientos, y de disfrutar además con ello, aumentando la seguridad en sí mismos y la autoestima.

"Como parte de la filosofía, la estética tiene por objeto el estudio de lo bello, lo sublime, lo ordenado, lo feo, lo gracioso. Aquí el ser humano logrará el equilibrio de la mente, cuerpo 
y espíritu, condiciones que elevaran su sensibilidad, imaginación y creatividad, fortaleciendo su personalidad para transformar y ser parte de una sociedad políticamente democrática, económicamente competitiva, socialmente justa y profundamente humanista, acorde con los avances tecnológicos y científicos". Carlos Germànico Toboada. Durante el periodo de edad que va desde los 0 a los 6 años, los niños aprenden haciendo algo que realmente les implica emocionalmente. Ese hacer se refiere a la acción efectiva, al pensamiento que actúa, es decir, a la posibilidad de pensar mediante el movimiento de descubrir y experimentar a través de la manipulación de los objetos y los materiales.

Los niños son por naturaleza artistas o inventores, poetas y músicos. La creación artística abre la vía para el aprendizaje de todo tipo de conocimientos y habilidades.

El arte no es solo una práctica o un producto, sino una manera particular de mirar el mundo, de sentir el mundo y de formar parte de ese mundo que el artista decide adoptar. La producción estética no es solo placer, sino una contribución a un desarrollo personal reflexivo que debe ser siempre uno de los objetivos fundamentales de la educación.

Los avances de la ciencia y la tecnología han hecho que en la sociedad actual se necesiten personas con ideas creativas, para responder a los retos que se plantean constantemente. La educación artística es una importante vía para desarrollar la capacidad de plantear soluciones divergentes, ya que es inherente a la propia naturaleza de las artes el fomentar, impulsar y dar cabida a la diversidad de las ideas, como forma de entender el sentido de su existencia.

Para la infancia y toda la comunidad educativa, las artes pueden introducir elementos extraordinarios en la construcción de las estructuras afectivas que organizan los ritmos colectivos y los rituales estéticos. Para ello, la escuela construye no solo un proyecto educativo, sino también cultural. En el ámbito de las artes, los niños y niñas aprenden a simbolizarse mediante la inmersión en los procesos de vida.

Mediante las metáforas de vida como juego simbólico compartido (el arte puede ser juego y actitud vital, abierto a la percepción, al conocimiento y a la reflexión), el niño y la niña acceden a lo simbólico como una conquista diaria que realizan a través de la educación artística como contexto de relación, acompañados de adultos e iguales, pues para construir el conocimiento se precisa no sólo de entender, sino entenderse en el mundo.

Así, las formas de expresión de las artes sitúan a la infancia en contextos significativos donde se da trascendencia a los acontecimientos y descubrimientos que los niños y niñas realizan, como una forma de visualización del proyecto de aprendizaje y son el escenario idóneo para reconocer sus capacidades de transformación. El educador facilita e identifica, de esta manera, la construcción de las vías de acceso a la experiencia estética infantil como hecho de vida y cultura. Todo ello mediante la inmersión en los procesos de vida.

\section{Materiales y Métodos}

Esta Investigación sigue una estrategia adecuada para llevar a la práctica este modelo que es " El método de investigación usado principalmente en la producción de conocimiento en las ciencias. Presenta diversas definiciones debido la complejidad de una exactitud en su conceptualización.", ya que permite interactuar en situaciones concretas y significativas, y estimula el " saber" el "saber hacer" y el "saber ser ", es decir, lo conceptual, lo procedimental y lo actitudinal.

La forma que tiene de comprender el mundo que le rodea, está muy alejada de la lógica del pensamiento adulto, sin embargo, la acción, la sensorialidad, la creatividad, la metáfora, el símbolo o la investigación son para la primera infancia la manera natural de desarrollar el aprendizaje.

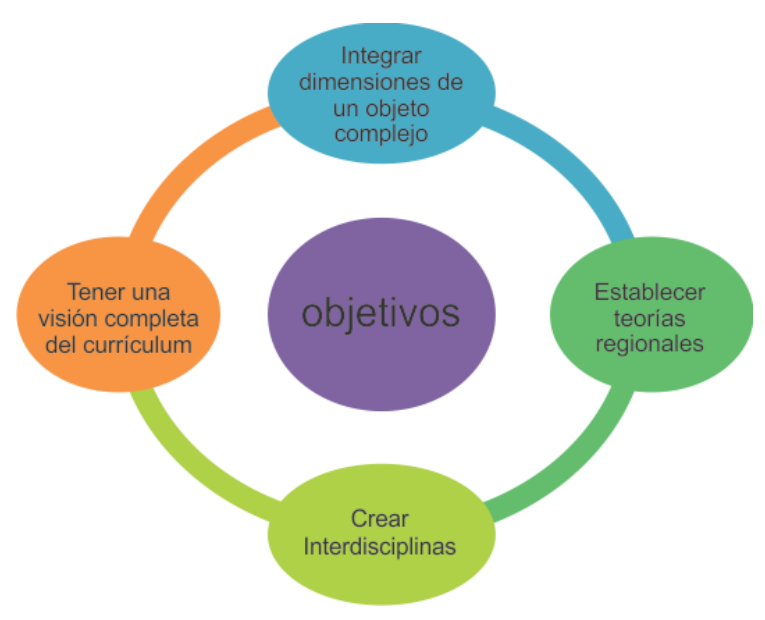

Figura 1.- Teoría Curricular.

Asimismo, la educación artística conecta con las necesidades y los intereses de la primera infancia, comprenden y comparte sus lenguajes comunicativos y expresivos. El arte es acción, 
movimiento, expresión, pensamiento, investigación, exploración y comunicación. Es entrar en contacto con uno mismo, con el espacio, el tiempo, los objetos y los demás.

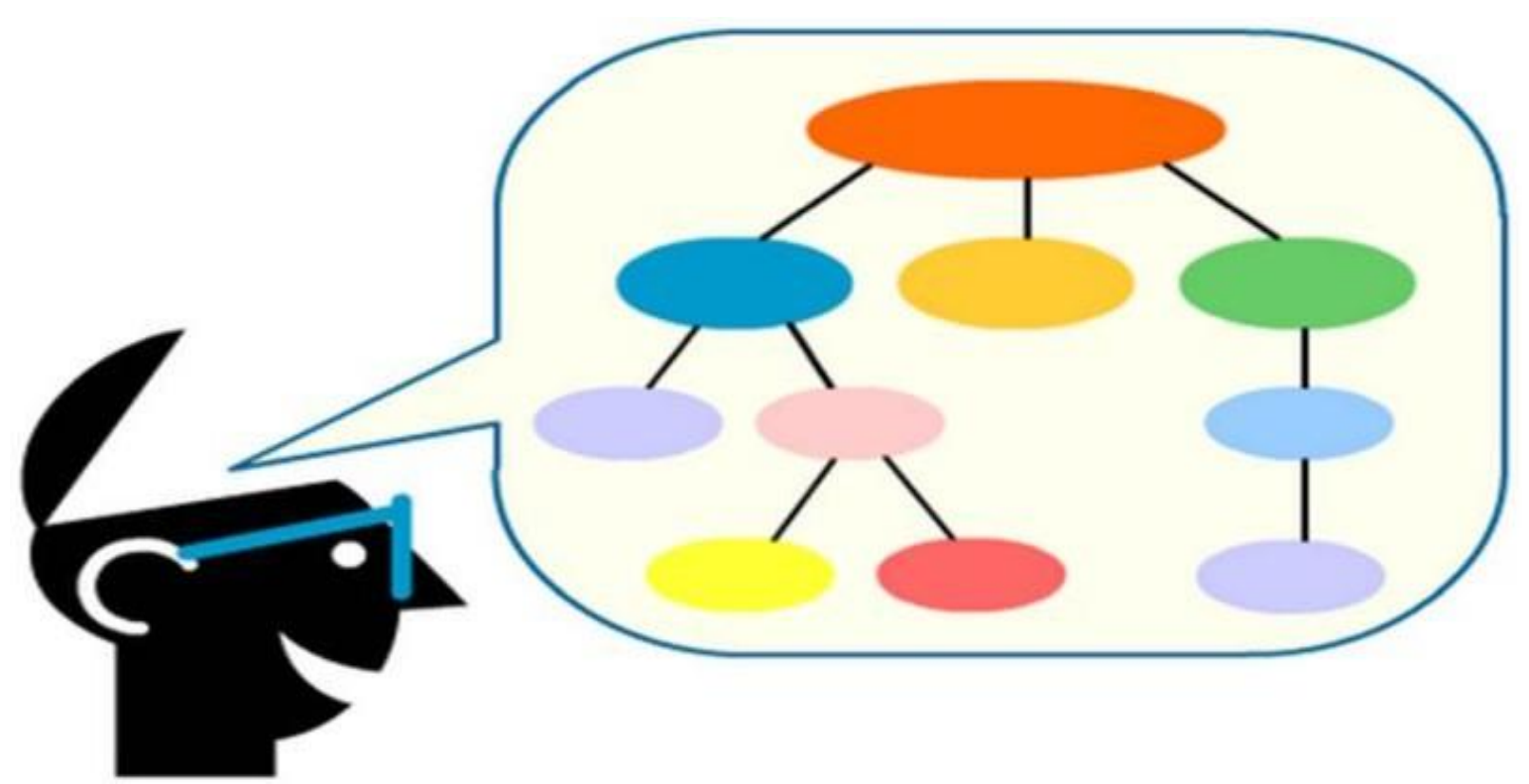

Figura 2.- Teoría del color.

\section{Características de este método}

Fáctico: Esto quiere decir que siempre se ciñe a los hechos.

Trasciende los hechos: Es cuando los investigadores no se conforman con las apariencias sino que buscan las causas $y$ efectos del fenómeno.

Se vale de la verificación empírica: Utiliza la comprobación de los hechos para formular respuestas del problema planteado y este está apoyado en la conclusión.

Es falible: No es infalible, puede perfeccionarse, a través de aportes utilizando nuevos procedimientos y técnicas.

No es autosuficiente: Necesita de algún conocimiento previo para luego reajustarse y elaborarse.

\section{Tipo de Investigación}

El presente es un trabajo netamente investigativo, por eso se ha considerado dos tipos de investigación: la Exploratoria y Descriptiva.

\section{Investigación Exploratoria}

Las ayudas exploratorias de la investigación determinan los mejores diseños de la investigación, método de la recolección de datos y selección de temas. Dada su naturaleza fundamental, la investigación exploratoria concluye a menudo que no existe un problema percibido realmente. Como manifestó Albert Einstein: "Si supiese qué es lo que estoy haciendo, no le llamaría investigación, ¿verdad? ".

\section{Investigación Descriptiva}

Comprende la descripción, registro, análisis e interpretación de la naturaleza actual, y la composición o procesos de los fenómenos. El enfoque se hace sobre conclusiones dominantes o sobre cómo una persona, grupo o cosa se conduce o funciona en el presente. La investigación descriptiva trabaja sobre realidades de hecho, y su característica fundamental es la presentación correcta.

\section{Instrumentos de la Investigación}

Por ser un proceso que conlleva necesariamente confiabilidad y validez, en el desarrollo del presente proyecto se utilizó instrumentos de investigación diseñados exclusivamente para este fin. Las técnicas utilizadas fueron: la Observación, la Ficha, la Encuesta, la Entrevista con su respectivo instrumento, el Cuestionario que contiene su respectiva Validez y Confiabilidad.

\section{Estadísticas}

La población total a investigar está compuesta 
por 72 personas del área de cultura estética de educación básica del Liceo Naval de Guayaquil, es decir, 2 Artistas, 10 docentes y 60 estudiantes, lo cual da un total de población de 70 personas a quienes se encuestará, y se entrevistará a 2 artistas, con lo cual la población será de 72 personas.

Según D’Onofre (1997) citado por Jiménez, Carlos (1999), Población "Es el conjunto agregado del número de elementos con caracteres comunes, en un espacio y tiempo determinado sobre los cuales se pueden realizar observaciones" (p.117).

\section{Discusión de Resultados}

Al comparar los resultados de las entrevistas realizadas a dos expertos en el área de Cultura Estética se obtiene el siguiente análisis de resultados:

> Los dos expertos consideran que en los colegios de Guayaquil no se conoce la importancia de la Cultura Estética en el ámbito educativo.

$>$ Como maestros con muchos años de experiencia, consideran que los contenidos de Dibujo Artístico se organizan de acuerdo al criterio de cada docente y de la experiencia con ciertas técnicas.

> Durante las clases de Dibujo Artístico no se explican las condiciones necesarias para realizar los ejercicios que se van a ejecutar, como en técnicas complejas como la acuarela, la tiza pastel e incluso anticipar las malas marcas de lápiz carboncillo que hay en el mercado.

> Las actividades interdisciplinarias con otras asignaturas no son suficientes, en todas las áreas básicas se necesita de la elaboración del apoyo visual, por parte de los estudiantes. como los carteles, maquetas y demás material de manipulación concreta.

$>$ Es bastante difícil entrar a un aula de clases y observar que el maestro maneja con sus estudiantes el término competencia, por lo general sólo se hace hincapié en la tarea, más no en lo que se quiere lograr con el ejercicio.

$>$ Es necesario que se reúnan los docentes semanalmente por áreas para planificar los temas a tratar en las clases, de esta manera los estudiantes podrán distinguir la secuencia en los temas impartidos e incluso cumplir con los materiales que se van a utilizar en las siguientes clases.
Los estudiantes detectan fácilmente la igualdad de contenido entre los paralelos, puesto que comparten sus experiencias con las técnicas que están aprendiendo e incluso en las pruebas al final de la unidad se puede notar la destreza de cada grupo y hasta dónde han llegado con los programas.

> Según las nuevas reformas de educación, se debe trabajar con Proyecto de Gestión de Aula que pueden ser de corto aliento o más extensos, abarcando hasta dos unidades.

Los estudiantes requieren de constantes actividades artísticas como exposiciones de trabajos, conferencias con artistas, visitas a museos y todos los soportes necesarios para que mejoren los contenidos en estudio.

> En las asignaturas básicas como Matemática, Lenguaje o Ciencias de la Naturaleza las editoriales están constantemente promocionando textos guías, donde se incluyen metodologías, talleres y competencias a desarrollar con los estudiantes. En el caso de Dibujo Artístico así mismo, cada maestro debe manejar una guía para dar un mayor respaldo a su asignatura.

\section{Análisis de Resultados de las Encuestas}

Al comparar los resultados de las encuestas realizadas a docentes, se obtiene el siguiente análisis de resultados.

> Los estudiantes demuestran deficiencias en el conocimiento significativo de la cultura estética en sus vidas. Así lo señala el 30\% de docentes y el $70 \%$ de estudiantes.

> Los docentes y estudiantes aclaran que nunca se han encontrado una secuencia lógica de los contenidos de Dibujo Artístico. Es lo que se infiere según lo indica el $50 \%$ de la encuesta aplicada a profesores y el 80 $\%$ de los estudiantes.

$>$ Falta que los docentes expliquen el adecuado uso del material de Dibujo Artístico en el aula de clases. Esto lo asegura el $50 \%$ de las personas consultadas mediante las encuestas realizadas y en los estudiantes el $90 \%$.

$>$ Los estudiantes no dominan términos importantes como lo es "competencia ". Es lo que se aprecia después de lo señalado por el $60 \%$ de docentes y el $70 \%$ de los 
estudiantes.

$>$ Los docentes nunca se reúnen a planificar sus clases. Es lo que se observa según lo indicado por el $70 \%$ de los docentes y el $33,3 \%$ de los estudiantes tienen criterios divididos.

$>$ No existe una nivelación de contenidos entre paralelos. Es lo que se interpreta después de las respuestas ofrecidas por el $50 \%$ de los docentes y el $70 \%$ de los estudiantes.

$>$ Los docentes no han tenido inconvenientes con la aplicación de proyectos de aula, aunque el $20 \%$ de los mismos indica que a veces sí se les han presentado inconvenientes para su ejecución. En cuanto a los estudiantes el $80 \%$ ha ejecutado Proyectos de Aula.

> Falta elaborar actividades artísticas con los estudiantes. Así lo confirma el $20 \%$ de los docentes encuestados. El $80 \%$ de los estudiantes aseguran que sí tienen actividades artísticas.

$>$ Todos los docentes encuestados demuestran su interés por aplicar una Guía Didáctica acerca del desarrollo de competencias como eje transversal de las otras asignaturas. Esto lo respalda el $80 \%$ de docentes que dieron su criterio, y en el caso de los estudiantes estos en un $90 \%$ desean más acceso a los ejemplos ejecutados en clase.

\section{Conclusiones}

En la presente investigación se explicitaron los fundamentos teóricos metodológicos que sustentan la formación pedagógica de los docentes, una vez realizado el análisis de los resultados obtenidos, es posible extraer las siguientes conclusiones:

- Existe una falencia notable en la secuencia de contenidos que se imparten entre los paralelos de educación básica del Liceo Naval de Guayaquil.

$>$ Es necesario que los docentes se tomen más tiempo en indicar la importancia y el adecuado manejo del material de Dibujo Artístico, según las técnicas que estén aplicando durante el desarrollo de la clase.

$>$ Los docentes deben planificar al inicio del año actividades extracurriculares e interdisciplinares que motiven a los estudiantes a asimilar mejor el arte, mediante visitas a museos, galerías $\mathrm{y}$ actividades culturales que se presenten en la ciudad.

Es importante que los estudiantes también conozcan de los lineamientos pedagógicos que los maestros están ejecutando en el aula, como lo es el concepto "competencia" y qué es lo que se quiere lograr con el ejercicio practicado.

Sobre la base de las conclusiones obtenidas, se recomienda presentar y reflexionar sobre los resultados a los docentes entrevistados.

A los docentes de Dibujo Artístico, se indica lo importante que el explicar con detalle el ejercicio que se esté ejecutado, el correcto uso del material con el que se está trabajando e indicar el significado de términos importantes que indican los logros obtenidos.

Se sugiere a los docentes del área de Cultura Estética que se reúnan cada cierto tiempo para organizar en primer lugar los contenidos a impartir, actividades extracurriculares e interdisciplinarias esto quiere decir, fomentar que los estudiantes relacionen lo que están aprendiendo con las otras asignaturas. También no está de más el organizar más programas culturales y artísticos dentro de la institución, muy aparte de las presentaciones de Proyectos de Aula.

> Se recomienda a los Directivos de cada Unidad Básica, se permita la realización de una Guía Didáctica para la asignatura de Dibujo Artístico, con el fin de apoyar a las demás asignaturas y también tener un soporte sobre los ejercicios ejecutados dentro del aula.

$>$ A los docentes de Cultura Estética, los expertos en arte recomiendan que con un documento que facilite la comprensión de los contenidos expuestos en Dibujo Artístico, se facilitará al analizar cualquier ejercicio que el estudiante no comprenda en el proceso de ejecución.

\section{Referencias}

1. Abad, J., Burset, S., Doménech, R., Duran, E., García Monte, C., y Treserras, M. 7 ideas clave la competencia cultural y artística. Barcelona: Graó. 2012.

2. Aguilar, M. La profesión académica como objeto de estudio. Antecedentes y referencias conceptuales. Revista de Ciencias Sociales, Vol. III (097): pp. 63- 7. Costa Rica. 2012 
3. Alsina, P., Giráldez, A. (Coor.) 7 Ideas Clave. La competencia cultural y artística. Colección Ideas Clave. Barcelona: Grao. 2012.

4. Altbach, P. El ocaso del gurú: la profesión académica en el tercer mundo. México: UNAM. 2008.

5. Díaz-Barriga, Ángel (coordinador). La investigación curricular en México 20022011, colección Estados del conocimiento, Ciudad de México: AnuiesComie. Entramado del Currículo y Desarrollo de Innovaciones en Educación. 2013.

6. Esquinas, F., Sánchez, M.(coor.) Dibujo 3 volúmenes. Colección formación del profesorado. Educación Secundaria: Barcelona Grao. 2011.
7. Giráldez, A. Formas de expresión y creaciones propias: competencia cultural y artística. Madrid: MEC. 2011.

8. Luengo Orcajo, F. Teoría y práctica de las competencias básicas. Graó, Barcelona. 2011.

9. Olmos, R. Competencia cultural y artística. Almería: Círculo rojo. 2012.

10. Sansot, S. Materiales didácticos potentes para la enseñanza. Ficha de circulación interna Cátedra Didáctica General, FCE, Universidad Nacional del Comahue. 2013.

11. Sobrino, M. Enseñar en tiempos mediáticos. Alemania: Editorial Académica Española. 2012 